\title{
Long non-coding RNA CCAT2 promotes epithelial-mesenchymal transition involving Wnt/ $\beta$-catenin pathway in epithelial ovarian carcinoma cells
}

\author{
BEIDI WANG, MEIMEI LIU, RUJIN ZHUANG，JING JIANG，JIAYIN GAO，HAO WANG, \\ HE CHEN, ZONGFENG ZHANG, YE KUANG and PEILING LI \\ Department of Obstetrics and Gynecology, The Second Affiliated Hospital of \\ Harbin Medical University, Harbin, Heilongjiang 150086, P.R. China
}

Received April 21, 2017; Accepted November 20, 2017

DOI: $10.3892 / \mathrm{ol} .2017 .7669$

\begin{abstract}
Long non-coding RNA colon cancer-associated transcript 2 (CCAT2) is dysregulated in a variety of types of human cancer. However, the role of CCAT2 in epithelial ovarian carcinoma (EOC) remains largely unknown. The aim of this study is to investigate the effect of CCAT2 on epithelial-mesenchymal transition (EMT) and related molecular mechanisms in epithelial ovarian cancer cells. In the current paper, we found that CCAT2 was significantly upregulated in EOC SKOV3, A2780 and HO8910 cell lines compared with the normal ovarian epithelial HUM-CELL-0088 cell line. Functional assays demonstrated that the knockdown of CCAT2 inhibited migration and invasion of EOC cells in vitro. Moreover, our results showed that silencing CCAT2 inhibited EMT by the upregulation of epithelial cadherin and downregulation of neural cadherin, zinc finger protein SNAI and Twist-related protein 1 in SKOV3 and A2780 cell lines. But, that was reversed by the treatment with lithium chloride ( $\mathrm{LiCl})$, by which the canonical Wnt/ $\beta$-catenin pathway could be activated. In addition, we further investigated the role of CCAT2 in the modulation of Wnt/ $\beta$-catenin signaling pathway. Our results revealed that knockdown of CCAT2 inhibited the expression of $\beta$-catenin and the activity of T-cell factor/lymphoid enhancer factor, acting as a key transcription factor of Wnt signaling pathway. Collectively, these results indicate that CCAT2 may promote EMT, at least partly through Wnt/ $\beta$-catenin signaling pathway in EOC cells. Thus, CCAT2
\end{abstract}

Correspondence to: Professor Peiling Li, Department of Obstetrics and Gynecology, The Second Affiliated Hospital of Harbin Medical University, 246 Xuefu Road, Harbin, Heilongjiang 150086, P.R. China

E-mail: peili0929@126.com

Key words: long non-coding RNA, colon cancer-associated transcript 2, epithelial-mesenchymal transition, epithelial ovarian carcinoma, Wnt signaling might play a critical role in EOC progression and serve as a valuable target for the treatment of ovarian cancer.

\section{Introduction}

Ovarian cancer is one of the most common gynecological malignancies and the incidence rate has been increasing annually (1). The most prevalent histological type is the epithelial ovarian carcinoma (EOC) which represents $85 \%$ of ovarian carcinoma (2). Despite advances in surgery and chemotherapy in the last decades, the overall survival of patients suffering from EOC is not satisfactory, with a 5-year survival rate of only $30 \%$ (3). Such a poor prognosis of patients with EOC has been largely correlated with tumor metastasis. Thus, it is urgently needed to understand the molecular mechanisms underlying ovarian cancer development and progression.

Epithelial-mesenchymal transition (EMT) is an important mechanism leading to invasion and metastasis of various cancers (4-6). Cells undergoing EMT lose the epithelial features and acquire some mesenchymal features, which is associated with the upregulation of $\mathrm{N}$-cadherin and downregulation of E-cadherin (6). EMT causes the failure of the intercellular connection that may facilitate the cancer cell to pass through basement membrane (7). E-cadherin is also known as cadherin $1(\mathrm{CDH} 1)$ and crucial for sustaining the polarity and structure of normal epithelial cell (8). Reduction of E-cadherin on the cell surface is clearly associated with poor overall survival of patients with EOC (9).

Colon cancer-associated transcript 2 (CCAT2), a novel lncRNA transcript reported by Ling et al, is located on 8q24 and highly overexpressed in microsatellite-stable colorectal cancer (10). Recently, Qiu et al reported that CCAT2 was highly expressed in non-small cell lung cancer (NSCLC). And the proliferation and invasion of NSCLC cell lines were inhibited in vitro after CCAT2 was silenced (11). Redis et al found that CCAT2 appeared to have higher expression in breast cancer tissue than in non-tumor tissue. Additionally, they observed that CCAT2 promoted cell migration and downregulated chemosensitivity to 5'FU in breast cancer (12). These studies indicated that CCAT2 acted as an oncogene in various human cancers. However, the detailed function and 
potential molecular mechanisms of CCAT2 in EOC are not fully understood and need to be investigated.

In the present study, we found that knockdown of CCAT2 inhibited EMT by upregulating E-cadherin and downregulating N-cadherin, Slug and Twist1 in SKOV3 and A2780. Furthermore, our results indicate that CCAT2 may exert its biological functions partly through Wnt/ $\beta$-catenin signaling pathway in the ovarian cancer cells. Therefore, the study revealed the important roles as well as the potential mechanisms of CCAT2 in ovarian cancer progression.

\section{Materials and methods}

Cell lines and cell culture. Two human EOC cell lines (SKOV3, A2780) were purchased from the Institute of Biochemistry and Cell Biology of Chinese Academy of Science (Shanghai, China). HO8910 was kindly provided by the Key Laboratory, Harbin Medical University, Ministry of Education (Heilongjiang, China). The EOC cell lines were cultured in RPMI-1640 medium (Corning Inc., Corning, NY, USA) containing $10 \%$ fetal bovine serum (FBS; Gibco Life Technologies, Carlsbad, CA, USA), penicillin (100 U/ml) and streptomycin $(100 \mathrm{mg} / \mathrm{ml})$ (both from Beyotime Institute of Biotechnology, Jiangsu, China). Human ovarian surface epithelial (HOSE) cell HUM-CELL-0088 was obtained from PriCells Biomedical Technology Co., Ltd. (Wuhan, China). HUM-CELL-0088 was cultured in DMEM (Corning, Inc.) with $10 \%$ FBS, penicillin $(100 \mathrm{U} / \mathrm{ml})$ and streptomycin $(100 \mathrm{mg} / \mathrm{ml})$. All of the above cells were maintained in a humidified $5 \% \mathrm{CO}_{2}$ incubator at $37^{\circ} \mathrm{C}$.

Total RNA extraction and reverse transcription-quantitative $P C R(R T-q P C R)$. Total RNA was isolated from cell lines using TRIzol reagent (Invitrogen, Auckland, New Zealand). RNA was reversely transcribed into cDNAs using a Transcriptor First Strand cDNA Synthesis kit (Roche Diagnostics, Indianapolis, IN, USA) according to the manufacturer's instructions. qPCR reactions performed using a qPCR System (Bio-Rad Laboratories, Inc., Hercules, CA, USA) and SYBR-Green PCR Master Mix (Roche Diagnostics). The primers used were: CCAT2, 5'-CCAGGCAATAACTGTGCAACTC-3' (sense) and 5'-ACTTACGTAGGGCATGCCAAA-3' (antisense); E-cadherin ( $C D H 1)$, 5'-TGCCCAGAAAATGAAAAAGG-3' (sense) and 5'-GTGTATGTGGCAATGCGTTC-3' (antisense); $\mathrm{N}$-cadherin ( $\mathrm{CDH} 2$ ), 5'-ACAGTGGCCACCTACAAAGG-3' (sense) and 5'-CCGAGATGGGGTTGATAATG-3' (antisense); Slug (SNAI2), 5'-TTCGGACCCACACATTACCT-3' (sense) and 5'-GCAGTGAGGGCAAGAAAAAG-3' (antisense); Twist1 (TWIST1), 5'-GGAGTCCGCAGTCTTACGAG-3' (sense) and 5'-TCTGGAGGACCTGGTAGAGG-3' (antisense); glyceraldehyde 3-phosphate dehydrogenase $(G A P D H), 5^{\prime}$-GAG TCAACGGATTTGGTCGT-3' (sense) and 5'-GACAAGCTT CCCGTTCTCAG-3' (antisense). Relative expression was calculated using the $2^{-\Delta \Delta \mathrm{Ct}}$ method. Each qPCR amplification was performed in triplicate to verify the results.

Small interfering RNAs (siRNAs) and transfection. For the study in vitro, SKOV3 and A2780 cells cultured on 6-well plate were transfected with either siRNAs targeting CCAT2 or negative controls (GeneChem, Shanghai, China) using lentivirus

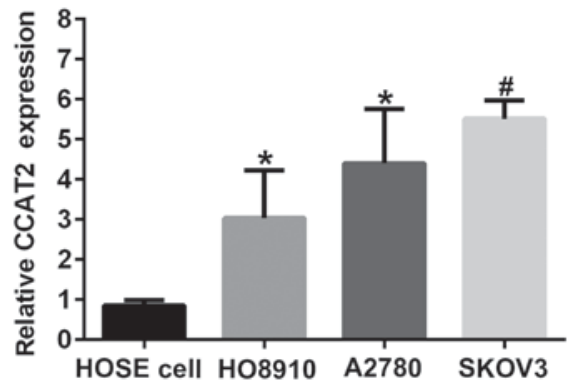

Figure 1. CCAT2 expression was upregulated in EOC SKOV3, A2780 and HO8910 cell lines compared with that in the HOSE HUM-CELL-0088 cell line. The data represent the mean \pm standard deviation of three independent experiments. ${ }^{*} \mathrm{P}<0.05$ and ${ }^{\#} \mathrm{P}<0.01$ compared to HOSE cell (Student's t-test). EOC, epithelial ovarian carcinoma; HOSE, human ovarian surface epithelial; CCAT2, colon cancer-associated transcript 2.

as vector (hU6-MCS-Ubiquitin-EGFP-IRES-puromycin) according to the instructions provided by the manufacturer. The siRNA sequences were as follows: 5'-AGGUGUAGCCAG AGUUAAUTT-3' (sense) and 5'-AUUAACUCUGGCUAC ACCUTT-3' (antisense). Cells were harvested for RT-qPCR and other experiments at $48 \mathrm{~h}$ after transfection.

Monolayer wound healing assay. Migration ability was measured using the wound healing assay. SKOV3 and A2780 were grown in 6-well plates. The cells were transfected with either siRNAs targeting CCAT2 or a negative control (NC). When cell reached $80 \%$ density, wounds were created in confluent cells using a $100 \mu \mathrm{l}$ pipette tip and then cells incubated in fresh medium for $48 \mathrm{~h}$. Three different locations were observed and photographed with an inverted phase contrast microscope (10X objective; Nikon, Japan, Tokyo). Each experiment was repeated at last three times.

Transwell invasion assay. Cell invasion assay was performed using Transwell chambers ( $8 \mu \mathrm{m}$ pore size; Corning Life Sciences, Tewksbury, MA, USA). For this assay, $100 \mu \mathrm{l}$ Matrigel (BD Biosciences, San Jose, CA, USA) was coated onto the upper chamber. The infected cells $\left(5 \times 10^{4}\right)$ were seeded in the upper chamber of the wells in $100 \mu \mathrm{l} \mathrm{FBS-free}$ medium, while the lower chambers were filled with $600 \mu \mathrm{l}$ $20 \%$ FBS medium. Following incubation for $24 \mathrm{~h}$, the top layer of the insert was scrubbed with a sterile cotton swab to remove any remaining cells. The invading cells on the bottom surface were fixed with methanol for $30 \mathrm{~min}$, stained with $0.1 \%$ crystal violet for $10 \mathrm{~min}$ and imaged using digital microscopy (10X objective; Nikon). Cell numbers were calculated in five random fields for each chamber at least and then the average value was calculated.

Western blot analysis. The harvested cells were disrupted in RIPA lysis buffer (Beyotime Institute of Biotechnology) containing protease inhibitor $[1 \mathrm{mM}$ phenylmethylsulfonyl fluoride (PMSF)]. After centrifugation, the supernatant fraction was harvested as the total cellular protein extract. The nuclear and cytoplasmic protein was extracted using Nuclear and cytoplasmic protein extraction kit (Beyotime Institute of Biotechnology) according to the manufacturer's instructions. The protein concentration was calculated using the Pierce 

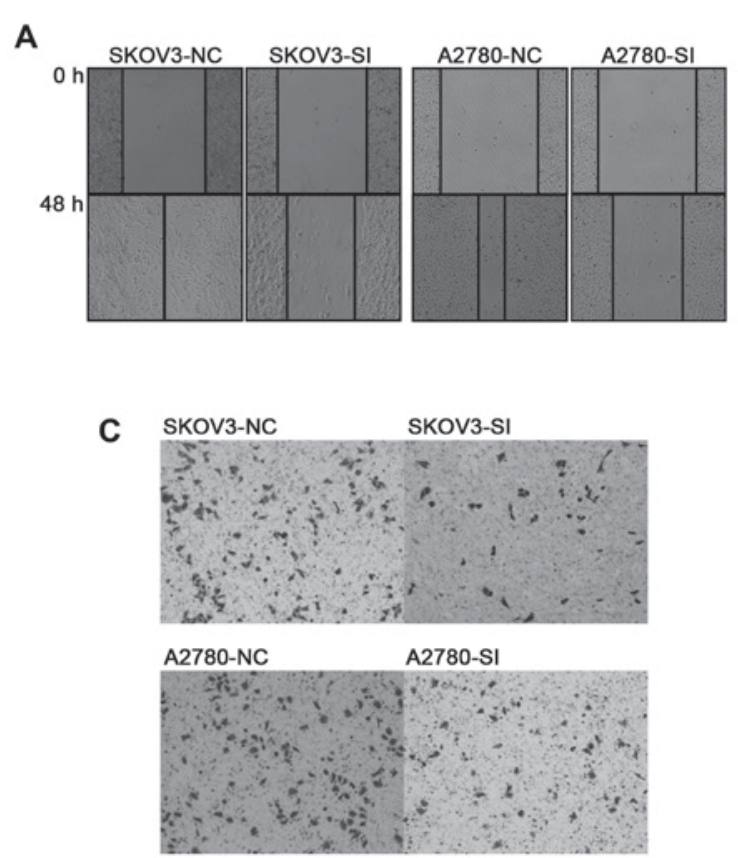
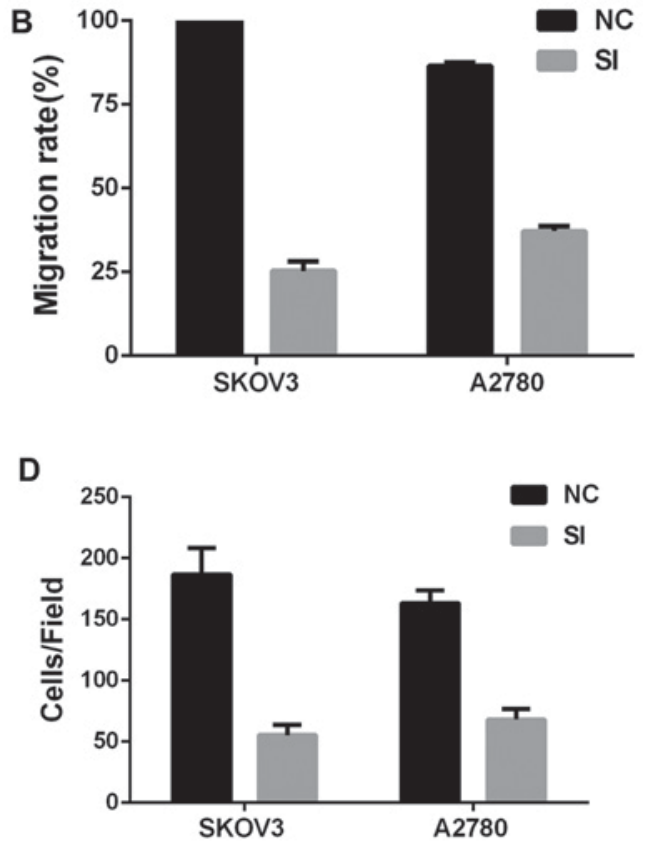

Figure 2. Silencing CCAT2 inhibited migration and invasion of EOC cells in vitro. (A) Representative images of the wound healing assay. (B) This assay showed that knockdown of CCAT2 reduced motility relative to NC cells. (C) Representative images of cell invasion was evaluated using a Matrigel invasion chamber. (D) Silencing CCAT2 inhibited invasion of SKOV3 and A2780 cells. The data represent the mean \pm SD of three independent experiments. P $<0.01$ compared to NC group (Student's t-test). NC, negative control; siRNAs, small interfering RNAs; CCAT2, colon cancer-associated transcript 2.

BCA protein assay kit (Beyotime Institute of Biotechnology). The cellular protein was separated by sodium dodecyl sulfate-polyacrylamide gel (SDS-PAGE) and transferred to polyvinylidene fluoride (PVDF) membranes (Invitrogen Life Technologies, Carlsbad, CA, USA). After blocking with $5 \%$ fat-free milk for $1 \mathrm{~h}$, the membrane was incubated with primary antibodies against E-cadherin (1:200 dilution, ab219332), N-cadherin (1:200 dilution, ab12221) (both from Abcam, Cambridge, MA, USA), Slug (1:500 dilution, C19G7), Twist1 (1:500 dilution, 46702), $\beta$-catenin (1:500 dilution, 9562) (all from Cell Signaling Technology, Inc., Danvers, MA, USA), $\alpha$-tubulin (1:2,000 dilution, ab7291), Lamin B1 (1:2,000 dilution, ab16048) and $\beta$-actin (1:2,000 dilution, ab8226) (all from Abcam) overnight at $4^{\circ} \mathrm{C}$ and then blots were washed 3 times in 1X Tris-Buffered Saline with $0.5 \%$ Tween (TBS-T), followed by incubation with an appropriate secondary antibody (1:5,000 dilution, ab6721 or ab6728; Abcam) for $1 \mathrm{~h}$. The blots were visualized using an enhanced chemiluminescence kit (Beyotime Institute of Biotechnology). $\alpha$-Tubulin, $\beta$-actin and lamin B1 were used as the loading control. Grayscale scanning for western blot analyses of three independent experiments was performed for quantitative analysis. The protein bands were analyzed using Gel-Pro Analyzer 6.3.0 software (Media Cybernetics, Rockville, MD, USA).

TOP-FLASH luciferase assay. Cells were transfected with $250 \mathrm{ng}$ of the TOP-FLASH reporter constructs together with $25 \mathrm{ng}$ of the Renilla luciferase vector. Luciferase activity was measured by the Dual Luciferase Reporter Assay System (Promega, Madison, WI, USA) $48 \mathrm{~h}$ after transfection. Renilla luciferase was used as the internal control, and TOP values were normalized to Renilla values. The TOP values were calculated and used as indicators of the endogenous level of Wnt signaling.

Statistical analysis. All of the statistical analyses were performed with SPSS 18.0 software (SPSS Inc., Chicago, IL, USA). Differences/correlations between two groups were assessed by the Student's t-test. P-values $<0.05$ were considered to indicate a statistically significant difference.

\section{Results}

CCAT2 is upregulated in ovarian cancer cell lines. To know about the profile of CCAT2 in EOC cell lines and HOSE cell, we characterized its expression by RT-qPCR in SKOV3, A2780, HO8910 and HUM-CELL-0088 (Fig. 1). CCAT2 expression was upregulated in ovarian cancer cell lines (SKOV3, A2780 and HO8910) compared with that in HOSE cell (HUM-CELL-0088). The results also showed that the expression level of CCAT2 in SKOV3 was the highest among three ovarian cancer cells, followed by that in A2780. SKOV3 and A2780 cell lines were chosen for subsequent experiments.

Silencing CCAT2 inhibited migration and invasion of EOC cell lines in vitro. To investigate whether CCAT2 plays a key role in facilitating cell migration and invasion, wound healing and invasion assays were performed. As was shown in Fig. 2, knockdown of CCAT2 significantly inhibited migration and invasion of EOC cells.

Knockdown of CCAT2 inhibits EMT in EOC cells. To test the hypothesis that CCAT2 may influence EMT in ovarian cancer cells, we examined a set of EMT marker genes, including 

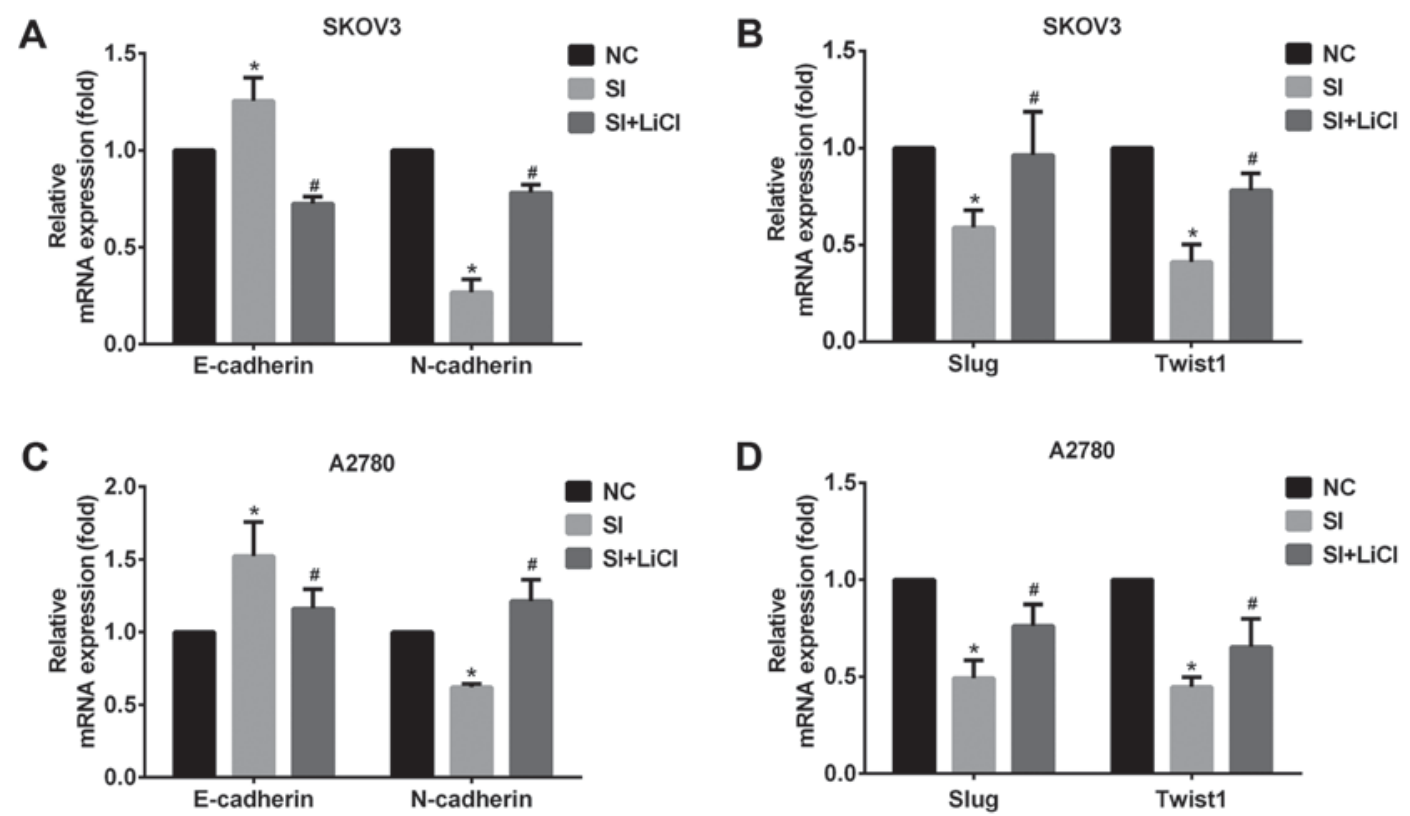

Figure 3. CCAT2 promotes EMT involving Wnt/ $\beta$-catenin signaling pathway in EOC cells. (A) Knockdown of CCAT2 increased the expression of E-cadherin and decreased that of N-cadherin, (B) Slug and Twist1 at the mRNA level in SKOV3 cells and (C) increased the expression of E-cadherin and decreased that of N-cadherin, (D) Slug and Twist1 at the mRNA level in A2780. But, that was reversed by the treatment with Wnt signaling activator LiCl (20 mM for $24 \mathrm{~h}$ ). The data represent the mean \pm standard deviation of three independent experiments. The values of the controls were set as 1 . "P<0.05 compared to NC group; "P<0.05 compared to SI group (Student's t-test). CCAT2, colon cancer-associated transcript 2; EMT, epithelial-mesenchymal transition; EOC, epithelial ovarian carcinoma; NC, negative control; siRNA, small interfering RNA; LiCl, lithium chloride; E-cadherin, epithelial cadherin; N-cadherin, neural cadherin; Slug, zinc finger protein SNAI; Twist1, Twist-related protein 1.

CDH1 (E-cadherin), CDH2 (N-cadherin) SNAI1 (Snail), SNAI2 (Slug) and Twist1. The data showed that knockdown of CCAT2 increased the expression of epithelial marker CDH1 but decreased that of mesenchymal marker $\mathrm{CDH} 2$ at the mRNA and protein levels (Figs. 3 and 4). Moreover, Slug and Twist1 were also downregulated when CCAT2 was silenced in EOC cells (Figs. 3 and 4). These results indicate that CCAT2 may be through the upregulation of Slug and Twist1 to promote EMT in ovarian cancer cells.

CCAT2 is involved in Wnt/ $\beta$-catenin signaling pathway. To better understand the detailed mechanisms of CCAT2 in ovarian cancer, we tested whether suppressing CCAT2 affected Wnt signaling pathway whose aberration played a key role in EOC development. The luciferase reporter system was employed in SKOV3 and A2780 cells. The results showed that silencing CCAT2 decreased TCF/LEF activity in the cells (Fig. 5A). We next measured the expression levels of $\beta$-catenin that is a key component of $\mathrm{Wnt} / \beta$-catenin signaling pathway. The data revealed that knockdown of CCAT2 decreased the expression of $\beta$-catenin both in the nucleus and cytoplasm at the protein level (Fig. 5C and D). In addition, we observed an obvious increase in CCAT2 expression after ovarian cancer cells were treated with lithium chloride ( $\mathrm{LiCl} ; 20 \mathrm{mM}$ for $24 \mathrm{~h}$ ) in order to activate canonical $\mathrm{Wnt} / \beta$-catenin signaling through the inhibition of glycogen synthase kinase $3 \beta$ (GSK3B) (Fig. 5B). These results strongly suggest that CCAT2 is involved in Wnt/ $\beta$-catenin signaling pathway and may elevate the signaling activity in EOC cells.

CCAT2 promotes EMT involving Wnt/ $\beta$-catenin signaling pathway. The above results showed that knockdown of CCAT2 inhibited the process of EMT (Figs. 3 and 4) and the activity of Wnt signaling (Fig. 5A and C). To further study the underlying mechanism by which CCAT2 induced EMT, ovarian cancer cells with knockdown of CCAT2 were treated with $\mathrm{LiCl}(20 \mathrm{mM})$ for $24 \mathrm{~h}$ to activate canonical Wnt $/ \beta$-catenin signaling. The results showed that it downregulated the expression of $\mathrm{CDH} 1$ and upregulated $\mathrm{CDH} 2$, Slug, Twist1 and CCAT2 (Figs. 3, 4 and 5B). Taken together, these data indicate that CCAT2 may promote EMT involving Wnt/ $\beta$-catenin signaling pathway in EOC cells.

\section{Discussion}

Recently, IncRNAs were discovered to be dysregulated in a variety of diseases, especially carcinomas (13-15). As a novel lncRNA, CCAT2 was initially reported for its overexpression in primary colorectal cancer and promotion of tumor growth, metastasis and chromosomal instability (10). Redis et al revealed that CCAT2 modulated colorectal cancer metabolism by interaction with the cleavage factor I (CFIm) complex in an allele-specific manner (16). Their study indicated that the mechanisms, underlying the regulation of metabolism, involved lncRNA, protein complexes, oncogenes and transcription factors. These findings provide some new and valuable clues to further explore the biological functions of CCAT2 in EOC, since the aberration of metabolism plays a really important role in ovarian cancer progression $(17,18)$. Wang et al reported that CCAT2 functioned as an oncogene in gastric cancer (GC) and was involved in GC progression (19). Huang et al found that CCAT2 expression was elevated in ovarian cancer tissues and cell lines. They also demonstrated that high CCAT2 level was significantly positively correlated 
A
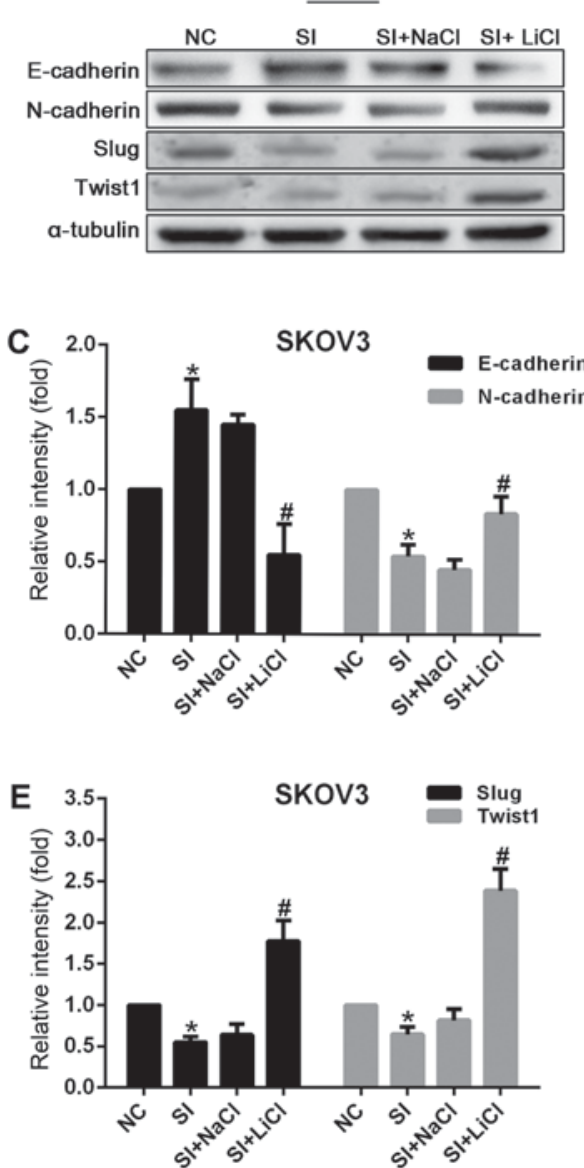

B
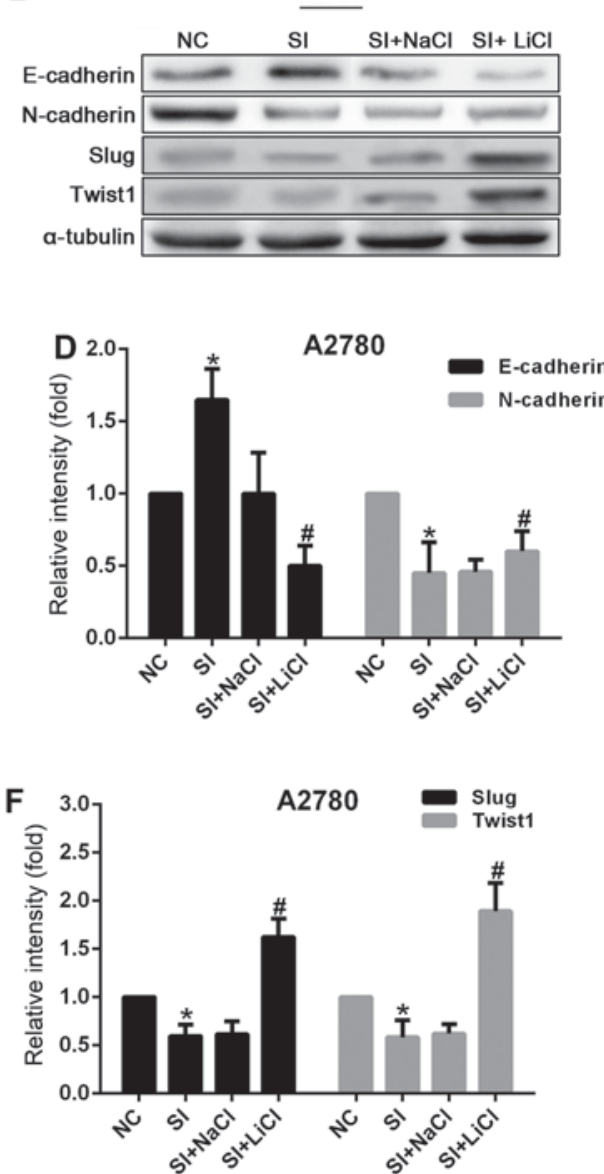

Figure 4. Knockdown of CCAT2 increased the expression of E-cadherin and decreased that of N-cadherin, Slug and Twist1 at the protein level in SKOV3 and A2780 cells. But, that was reversed by the treatment with Wnt signaling activator $\mathrm{LiCl}(20 \mathrm{mM})$ for $24 \mathrm{~h}(20 \mathrm{mM} \mathrm{NaCl}$ as control). Representative images of western blot analysis for (A) SKOV3 and (B) A2780 are presented. The data represent the mean \pm standard deviation of three independent experiments. The values of the controls were set as $1 .{ }^{*} \mathrm{P}<0.05$ compared to NC group; ${ }^{\prime} \mathrm{P}<0.05$ compared to the SI+NaCl group (Student's t-test). CCAT2, colon cancer-associated transcript 2; LiCl, lithium chloride; NC, negative control; siRNA, small interfering RNA; E-cadherin, epithelial cadherin; N-cadherin, neural cadherin; Slug, zinc finger protein SNAI; Twist1, Twist-related protein 1.

A

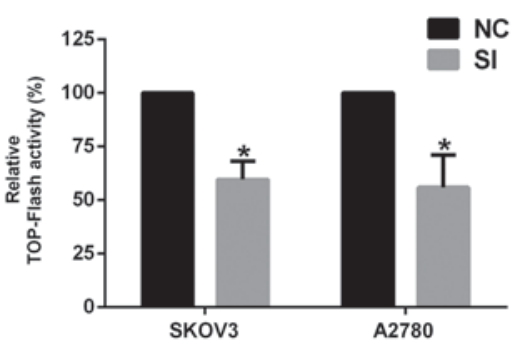

C

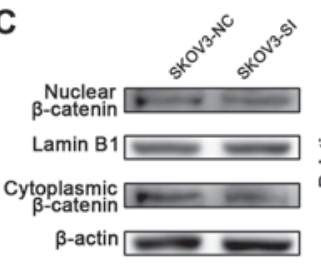

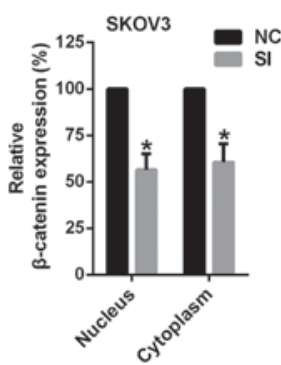

B

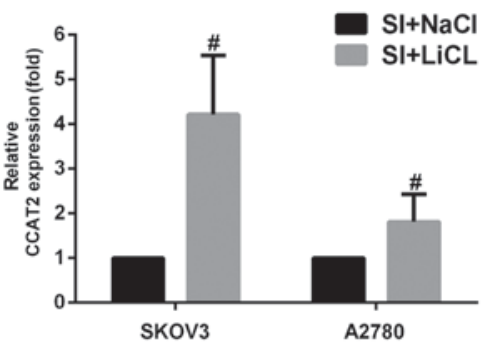

D

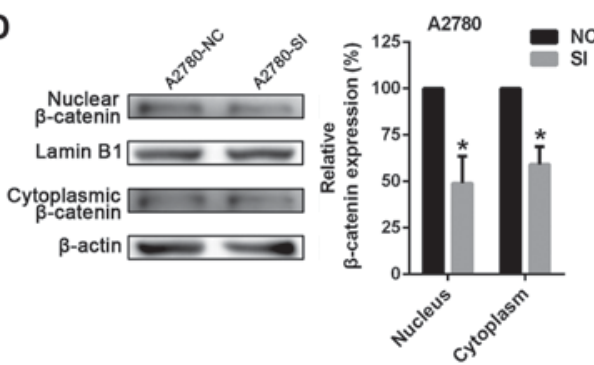

Figure 5. CCAT2 is involved in Wnt/ $\beta$-catenin signaling pathway. (A) TOP-FLASH luciferase reporter assay showed that knockdown of CCAT2 downregulated TCF/LEF activity in SKOV3 and A2780. (B) The expression of CCAT2 was increased by LiCl treatment (20 mM, for $24 \mathrm{~h})$ to further activate canonical Wnt/ $\beta$-catenin signaling. (C) Knockdown of CCAT2 decreased the expression of $\beta$-catenin both in the nucleus and cytoplasm at the protein level in SKOV3 and (D) A2780 cells. The data represent the mean \pm SD of three independent experiments. The values of the controls were set as $1 .{ }^{*} \mathrm{P}<0.05$ compared to NC group; " $\mathrm{P}<0.05$ compared to $\mathrm{SI}+\mathrm{NaCl}$ group (Student's t-test). CCAT2, colon cancer-associated transcript 2; NC, negative control; siRNA, small interfering RNA; LiCl, lithium chloride; TOP-FLASH, TCL/LEF-Firefly luciferase; $\mathrm{LiCl}$, lithium chloride. 
with FIGO stage, tumor grade and distant metastasis of ovarian cancer (20). However, the molecular mechanisms by which CCAT2 promotes EOC metastasis and progression are not fully understood. In the current study, we investigated the effect of CCAT2 on EMT and related molecular mechanisms in epithelial ovarian cancer cells. Our results demonstrated that CCAT2 played a functional role in promoting EMT of EOC cells.

As a significant biological process, EMT involves a change from an epithelial to a mesenchymal phenotype in epithelial cell. Downregulation of E-cadherin and upregulation of $\mathrm{N}$-cadherin are the hallmarks of EMT (21). N-cadherin is a mesenchymal feature, while E-cadherin is an epithelial adhesion glycoprotein whose decrease could make the junctions between epithelial cells dissolved and facilitate migratory and aggressive behavior (22). Previous studies demonstrated that the expression of E-cadherin was reduced in various kinds of invasive tumors including epithelial ovarian carcinoma (23-25). In the present study, we observed that silencing CCAT2 inhibited cell migration and invasion, as well as EMT of EOC cells. Furthermore, we found that Slug and Twistl were both downregulated at the mRNA and protein levels after CCAT2 was knocked down. As is known, Slug and Twistl could facilitate the process of EMT by modulating E-cadherin and N-cadherin. Therefore, these findings strongly suggest that Slug and Twist1 may be the major targets by which CCAT2 modulates EMT of ovarian cancer cells.

Canonical Wnt/ $\beta$-catenin signaling pathway is important for the induction of EMT $(26,27)$. Studies demonstrated that the aberrant signaling of Wnt pathway played a key role in EMT during colorectal cancer progression (28). Previous research has shown that the suppression of Wnt signaling inhibited cell migration and promoted cell adhesion in ovarian cancer cells, which was attributed to the inhibition of EMT (29). By virtue of immunoprecipitation assay targeting TCF7L2 (protein) and CCAT2 (RNA), Ling et al found that CCAT2 might markedly modulate the downstream genes of Wnt signaling pathway by combining with TCF7L2 and increasing its transcriptional activity in colon cancer cells (10). In this study, we detected that knockdown of CCAT2 inhibited the activity of TCF/LEF in SKOV3 and A2780. Moreover, our investigation revealed that silencing CCAT2 decreased the expression of $\beta$-catenin both in the nucleus and cytoplasm at the protein level. These results demonstrated that knockdown of CCAT2 inhibited the activity of Wnt signaling in EOC cells, which was in line with the finding in glioma and breast cancer $(30,31)$.

The above results revealed that knockdown of CCAT2 inhibited the process of EMT and the activity of Wnt signaling. To further study the underlying mechanisms by which CCAT2 induced EMT, cells with knockdown of CCAT2 were treated with $\mathrm{LiCl}$ to activate canonical $\mathrm{Wnt} / \beta$-catenin signaling. We found that $\mathrm{LiCl}$ treatment not only reversed the effect of silencing CCAT2 on EMT, but also greatly enhanced CCAT2 expression. These results suggest that CCAT2 may promote the process of EMT, at least partly, through Wnt/ $\beta$-catenin signaling pathway in epithelial ovarian carcinoma cells. The data also indicate there might be a complex feedback loop between CCAT2 and Wnt signaling that needs to be further explored in the future.
In conclusion, the present study advances the understanding of the molecular mechanisms by which CCAT2 facilitates EMT. Our findings suggest that CCAT2 may play an important role in EOC progression. Thus, CCAT2 may serve as a potential target for the treatment of ovarian cancer.

\section{Acknowledgements}

The authors thank Yufei Jiao (Department of Pathology, the Second Affiliated Hospital of Harbin Medical University), Xianli Zhou (Department of Ultrasound Medicine, the Second Affiliated Hospital of Harbin Medical University) for providing general support for this study. This study was supported by Harbin Medical University.

\section{References}

1. Menon U, Gentry-Maharaj A and Jacobs I: Ovarian cancer screening and mortality. JAMA 306: 1544-1545, 2011.

2. Jelovac D and Armstrong DK: Recent progress in the diagnosis and treatment of ovarian cancer. CA Cancer J Clin 61: 183-203, 2011.

3. Bast RC Jr, Hennessy B and Mills GB: The biology of ovarian cancer: New opportunities for translation. Nat Rev Cancer 9: 415-428, 2009.

4. Fan WH, Du FJ, Liu XJ and Chen N: Knockdown of FRAT1 inhibits hypoxia-induced epithelial-to-mesenchymal transition via suppression of the $\mathrm{Wnt} / \beta$-catenin pathway in hepatocellular carcinoma cells. Oncol Rep 36: 2999-3004, 2016.

5. Mo D, Yang D, Xiao X, Sun R, Huang L and Xu J: miRNA-145 suppresses lung adenocarcinoma cell invasion and migration by targeting N-cadherin. Biotechnol Lett: 701-710, 2017.

6. Lim J and Thiery JP: Epithelial-mesenchymal transitions: Insights from development. Development 139: 3471-3486, 2012.

7. Ramis-Conde I, Chaplain MA, Anderson AR and Drasdo D: Multi-scale modelling of cancer cell intravasation: The role of cadherins in metastasis. Phys Biol 6: 016008, 2009.

8. van Roy F: Beyond E-cadherin: Roles of other cadherin superfamily members in cancer. Nat Rev Cancer 14: 121-134, 2014.

9. Faleiro-Rodrigues C, Macedo-Pinto I, Pereira D and Lopes CS: Prognostic value of E-cadherin immunoexpression in patients with primary ovarian carcinomas. Ann Oncol 15: 1535-1542, 2004.

10. Ling H, Spizzo R, Atlasi Y, Nicoloso M, Shimizu M, Redis RS, Nishida N, Gafà R, Song J, Guo Z, et al: CCAT2, a novel noncoding RNA mapping to $8 \mathrm{q} 24$, underlies metastatic progression and chromosomal instability in colon cancer. Genome Res 23: 1446-1461, 2013.

11. Qiu M, Xu Y, Yang X, Wang J, Hu J, Xu L and Yin R: CCAT2 is a lung adenocarcinoma -specific long non-coding RNA and promotes invasion of non-small cell lung cancer. Tumour Biol 35: 5375-5380, 2014.

12. Redis RS, Sieuwerts AM, Look MP, Tudoran O, Ivan C, Spizzo R, Zhang X, de Weerd V, Shimizu M, Ling H, et al: CCAT2, a novel long non-coding RNA in breast cancer: Expression study and clinical correlations. Oncotarget 4: 1748-1762, 2013.

13. Crea F, Clermont PL, Parolia A, Wang Y and Helgason CD: The non-coding transcriptome as a dynamic regulator of cancer metastasis. Cancer Metastasis Rev 33: 1-16, 2014.

14. Richards EJ, Permuth-Wey J, Li Y, Chen YA, Coppola D, Reid BM, Lin HY, Teer JK, Berchuck A, Birrer MJ, et al: A functional variant in HOXA11-AS, a novel long non-coding RNA, inhibits the oncogenic phenotype of epithelial ovarian cancer. Oncotarget 6: 34745-34757, 2015.

15. Qiu JJ, Lin YY, Ye LC, Ding JX, Feng WW, Jin HY, Zhang Y, Li Q and Hua KQ: Overexpression of long non-coding RNA HOTAIR predicts poor patient prognosis and promotes tumor metastasis in epithelial ovarian cancer. Gynecol Oncol 134: $121-128,2014$.

16. Redis RS, Vela LE, Lu W, Ferreira de Oliveira J, Ivan C, Rodriguez-Aguayo C, Adamoski D, Pasculli B, Taguchi A and Chen Y, et al: Allele-specific reprogramming of cancer metabolism by the long non-coding RNA CCAT2. Mol Cell 61: 520-534, 2016. 
17. Nieman KM, Kenny HA, Penicka CV, Ladanyi A Buell-Gutbrod R, Zillhardt MR, Romero IL, Carey MS, Mills GB, Hotamisligil GS, et al: Adipocytes promote ovarian cancer metastasis and provide energy for rapid tumor growth. Nat Med 17: 1498-1503, 2011.

18. Anderson AS, Roberts PC, Frisard MI, Hulver MW and Schmelz EM: Ovarian tumor-initiating cells display a flexible metabolism. Exp Cell Res 328: 44-57, 2014

19. Wang YJ, Liu JZ, Lv P, Dang Y, Gao JY and Wang Y: Long non-coding RNA CCAT2 promotes gastric cancer proliferation and invasion by regulating the E-cadherin and LATS2. Am J Cancer Res 6: 2651-2660, 2016.

20. Huang S, Qing C, Huang Z and Zhu Y: The long non-coding RNA CCAT2 is up-regulated in ovarian cancer and associated with poor prognosis. Diagn Pathol 11: 49, 2016.

21. Thiery JP, Acloque H, Huang RY and Nieto MA: Epithelial-mesenchymal transitions in development and disease. Cell 139: 871-890, 2009.

22. Thiery JP and Sleeman JP: Complex networks orchestrate epithelial-mesenchymal transitions. Nat Rev Mol Cell Biol 7: 131-142, 2006.

23. Onder TT, Gupta PB, Mani SA, Yang J, Lander ES and Weinberg RA: Loss of E-cadherin promotes metastasis via multiple downstream transcriptional pathways. Cancer Res 68: 3645-3654, 2008.

24. Sawada K, Mitra AK, Radjabi AR, Bhaskar V, Kistner EO, Tretiakova M, Jagadeeswaran S, Montag A, Becker A, Kenny HA, et al: Loss of E-cadherin promotes ovarian cancer metastasis via alpha 5-integrin, which is a therapeutic target. Cancer Res 68: 2329-2339, 2008.
25. Poser I, Domı́nguez D, Herreros AG, Varnai A, Buettner R and Bosserhoff AK: Loss of E-cadherin expression in melanoma cells involves up-regulation of the transcriptional repressor Snail. J Biol Chem 276: 24661-24666, 2001.

26. Arend RC, Londono-Joshi AI, Straughn Jr JM and Buchsbaum DJ: The Wnt $/ \beta$-catenin pathway in ovarian cancer: A review. Gynecol Oncol 131: 772-779, 2001

27. Lamouille S, Xu J and Derynck R: Molecular mechanisms of epithelial-mesenchymal transition. Nat Rev Mol Cell Biol 15: 178-196, 2014.

28. Gonzalez DM and Medici D: Signaling mechanisms of the epithelial-mesenchymal transition. Sci Signal 7: re8, 2014.

29. Ford CE, Jary E, Ma SS, Nixdorf S, Heinzelmann-Schwarz VA and Ward RL: The Wnt gatekeeper SFRP4 modulates EMT, cell migration and downstream Wnt signaling in serous ovarian cancer cells. PLoS One 8: e54362, 2013.

30. Hua G, Hu G, Yang Q, Zhang P, Kuang W, Zhu X and Wu L: Knockdown of long non-coding RNA CCAT2 suppressed proliferation and migration of glioma cells. Oncotarget 7: 81806-81814, 2016.

31. Cai Y, He J and Zhang D: Long noncoding RNA CCAT2 promotes breast tumor growth by regulating the Wnt signaling pathway. Onco Targets Ther 8: 2657-2664, 2015. 